\title{
Impacto de eventos de cizalladura severa en el aeropuerto de Gran Canaria
}

\author{
David Suárez Molina ${ }^{1}$ (dsuarezm@aemet.es) \\ Javier Fernández Villares ${ }^{1}$ (jfernandezv@aemet.es) \\ Sergio Fernández González² (sfernandezg@aemet.es)
}

${ }^{1}$ AEMET / Delegación Territorial en Canarias

${ }^{2}$ AEMET / Dirección de Producción e Infraestructuras

\begin{abstract}
RESUMEN
La cizalladura es el cambio en la dirección y/o intensidad del viento en un plano y en una distancia espacial corta. Se produce cuando capas de aire adyacentes tienen una acusada diferencia entre sus velocidades respectivas.

Existen numerosas causas que pueden ocasionar cizalladura como por ejemplo: microrreventones (microbursts) y frentes de racha inducidos por tormentas, interacción del viento sobre terreno montañoso, brisa marina, chorro de niveles bajos, superficies frontales, etc.

En este estudio se analizarán las dos situaciones más extremas de cizalladura en niveles bajos que ha sufrido el aeropuerto de Gran Canaria (GCLP) en los últimos años. Para ello se combinarán salidas de modelos de predicción numérica (NWP) con datos de observación. Durante estos episodios ( 26 de abril de 2017 y 28 de febrero de 2018) se produjeron 35 aterrizajes frustrados, que ocasionaron numerosos desvíos a aeropuertos vecinos e innumerables pérdidas económicas a compañías aéreas.
\end{abstract}

PALABRAS CLAVE: cizalladura; windshear; predicción aeronáutica; modelización; frustada.

\section{INTRODUCCIÓN}

La cizalladura en niveles bajos es uno de los peligros más críticos de la aviación (MAzon et al., 2018). Detectarla de forma precisa, así como ser capaces de alertar a los usuarios, debe ser el objetivo principal para garantizar la seguridad en vuelo (KESSLER, 1990). El fenómeno de cizalladura puede presentarse en cualquier nivel de la atmósfera, pero esta tiene un especial interés por debajo de 2000 o 3000 pies AGL (sobre el nivel del terreno), cizalladura a baja altura (Low Level Windshear (LLWS)), donde puede afectar a las maniobras de despegue y aterrizaje.

La cizalladura es la causa fundamental de la turbulencia. Esta puede ser vertical, horizontal o ambas, o ser debida únicamente a cambios en la componente vertical del viento (originada por corrientes verticales ascendentes o descendentes) (GoNZÁLEZ, 2005).

La cizalladura del viento a baja altura supone un peligro potencial cuando las variaciones en la intensidad del viento causan un cambio súbito de la sustentación de las aeronaves haciendo difícil su gobernabilidad. 
El aeropuerto de Gran Canaria (GCLP) está situado a 24 metros de altitud en la zona este de la isla. Las pistas se orientan 030-210 grados de acuerdo al flujo dominante, los vientos alisios, que soplan del nordeste con una frecuencia del $90 \%$ en verano y del $50 \%$ el resto del año (FonT, 1956). El complejo relieve de la isla provoca que el flujo se perturbe y se genere cizalladura inducida por la orografía en numerosas ocasiones a lo largo del año.

Se han analizado más de 15000 notificaciones ATIS (Automatic Terminal Information Service) correspondientes a cizalladura en niveles bajos en el aeropuerto de Gran Canaria (GCLP), durante el periodo comprendido entre el 1 de enero de 2015 al 31 de mayo de 2018.

Los resultados del estudio estadístico que incluyen cizalladura y/o viento en cola relacionado con el viento presente en el aeropuerto GCLP se muestran en la figura 1, en la que se han analizado los mensajes ATIS, estudiándose por separado:

- Notificaciones ATIS que incluyen windshear(WS)

- Notificaciones ATIS que incluyen tail wind (TW)

- Notificaciones ATIS que incluyen windshear (WS) y tail wind (TW)

Se observa que el $89 \%$ de los casos de cizalladura (WS) se presenta con vientos en el aeropuerto del primer y cuarto cuadrantes, y de este valor, el $86 \%$ corresponde a las direcciones de viento comprendidas entre $310^{\circ}-030^{\circ}$, lo que corrobora lo comentado anteriormente de la mayor presencia de cizalladura con alisio de acentuada componente norte $(\mathrm{N}-\mathrm{NW})$.

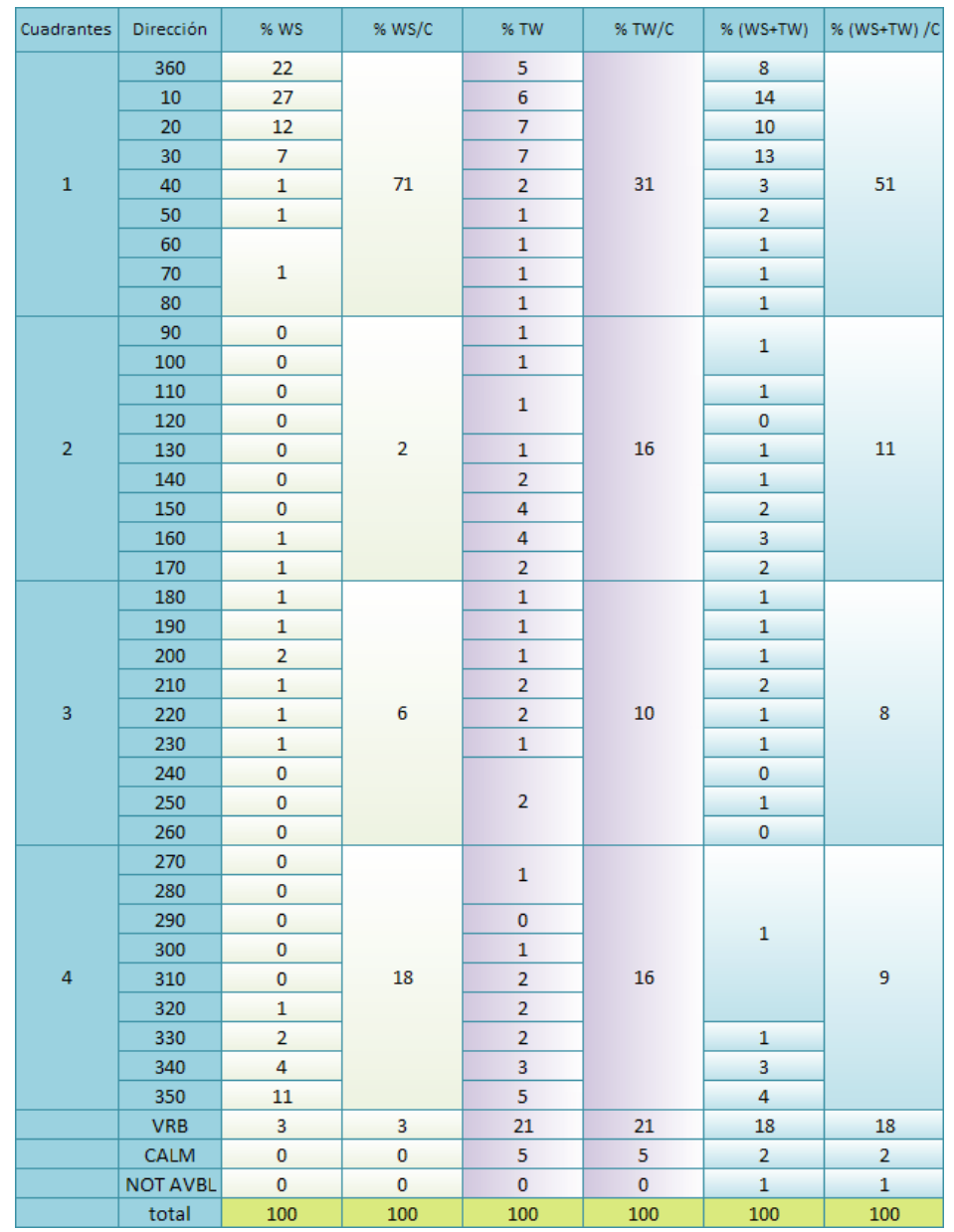

Figura 1. Tabla que muestra los porcentajes de notificaciones de cizalladura en función de la dirección del viento.
Para los vientos de componente sur, el $9 \%$ de los ATIS con cizalladura se asocian con vientos entre $160^{\circ}-230^{\circ}$, por lo que podemos concluir que en el $95 \%$ de los casos que se notifica cizalladura el flujo es de las direcciones:

$$
\begin{aligned}
& \text { - } 310^{\circ}-030^{\circ} \rightarrow 86 \% \\
& \text { - } 160^{\circ}-230^{\circ} \rightarrow 9 \%
\end{aligned}
$$

Los datos señalan que la cizalladura es principalmente inducida por el terreno. Las frecuencias por intensidad de viento de estas direcciones predominantes y, en general, los eventos de cizalladura aumentan con la intensidad del flujo.

Referente a las operaciones frustradas, en su gran mayoría aterrizajes, la figura 2 muestra los resultados del estudio del periodo comprendido entre abril de 2015 y marzo de 2018, y se observan unos máximos bien marcados en determinados meses, correspondiendo en los tres casos a situaciones de viento del SW, con un gran número de operaciones frustradas en un solo día. 


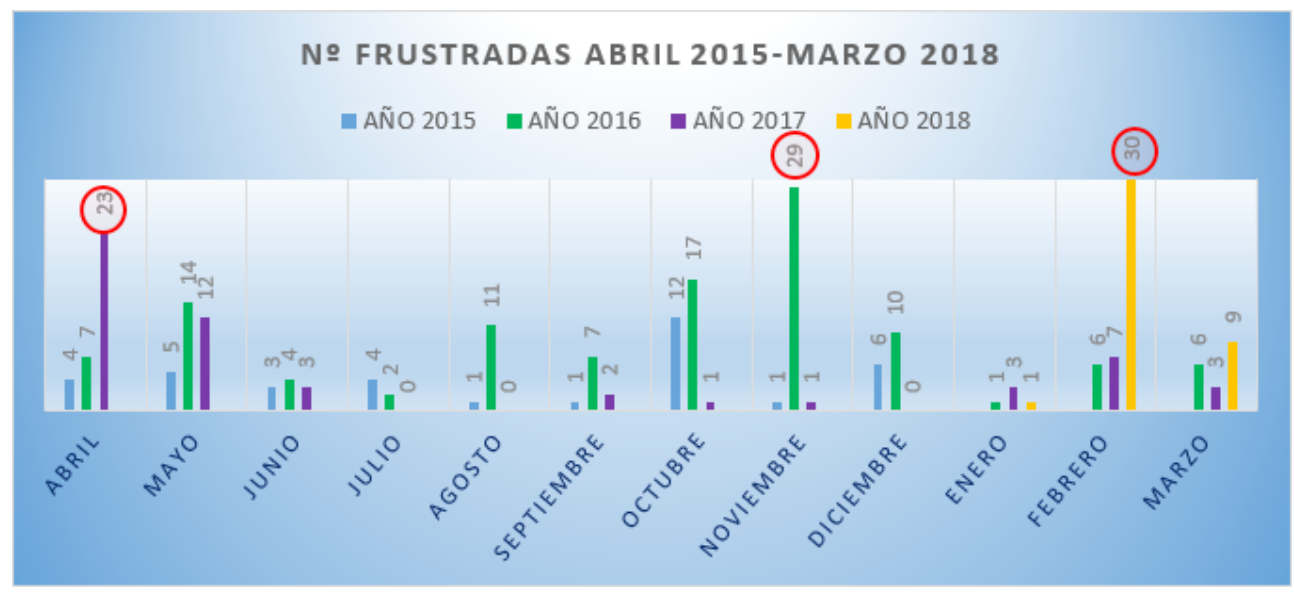

Figura 2. Número de operaciones frustradas entre el periodo comprendido entre abril de 2015 y marzo 2018.

\section{ANÁLISIS DE DOS EVENTOS DE CIZALLADURA SEVERA}

A pesar de su menor incidencia, los eventos más desfavorables de cizalladura ocurren precisamente en situaciones donde el flujo es del SW y W, con capas de inversión elevadas (e incluso inexistentes) que permiten al flujo sobrepasar los obstáculos orográficos y acentuar esa cizalladura inducida por el propio terreno.

La situación meteorológica (figura 3) corresponde con una borrasca al W o NW de Canarias, quedando las islas en el sector de vientos del SW de la baja, como se observa en los siguientes mapas correspondientes a los dos eventos de cizalladura que mayor número de operaciones frustradas han ocasionado en los últimos años.

En la figura 3, en la que se expone el análisis para el nivel de superficie del modelo del Centro Europeo de Predicción a Medio Plazo (ECMWF, por sus siglas en inglés) y se representa la presión en superficie en $\mathrm{hPa}$ y la temperatura sombreada en el nivel de $850 \mathrm{hPa}$, se observa que la situación sinóptica ambos días era similar aunque con diferencias en la posición y profundidad de los sistemas de baja presión. Por un lado el día 28 de febrero de 2018 la baja se situaba al norte de Madeira y este de Azores con un mínimo de presión de $980 \mathrm{hPa}$, que generaba vientos del oeste intensos sobre el archipiélago canario. En la situación correspondiente al día 26 de abril de 2017, la baja se situaba al noroeste de Madeira, con su centro más cercano a Canarias y con una presión de $1006 \mathrm{hPa}$ que producía un viento del suroeste menos intensos que en la situación anterior.

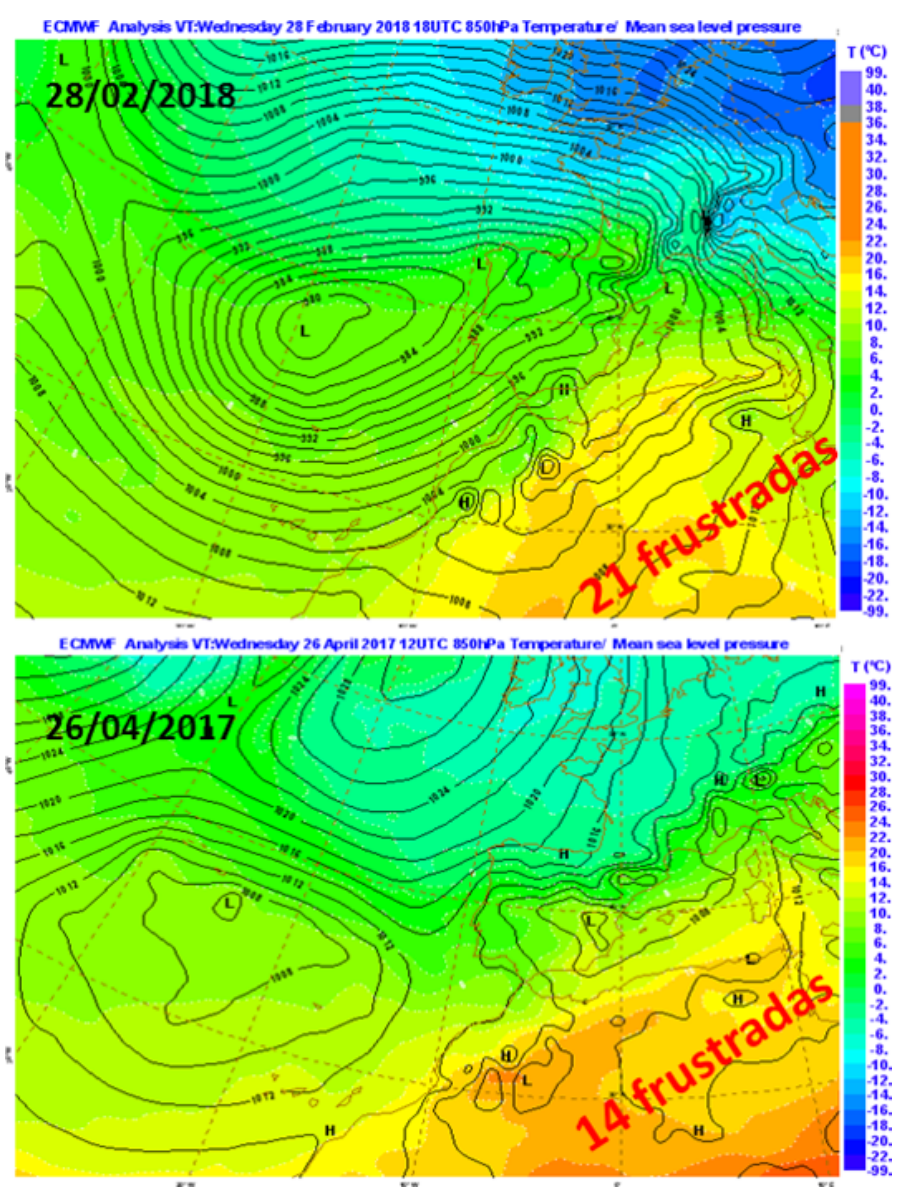

Figura 3. Análisis de superficie del modelo del Centro Europeo correspondiente a los días 28 de febrero de 2018 y 26 de abril de 2017. 
La cizalladura en niveles bajos puede estar directamente influenciada por la orografía, y por ello se ha decidido utilizar modelos de mayor resolución espaciotemporal, como es el caso del modelo HarmonieArome. Este modelo tiene una resolución espacial de 2,5 $\mathrm{km}$ y es de utilidad para simular procesos de mesoescala. Hay que tener en cuenta que la turbulencia $y$, por lo tanto, la cizalladura es un fenómeno de microescala, y esto supone una clara limitación al problema de la predicción de este tipo de fenómenos. En la figura 4 se muestra el viento a 10 metros en nudos previsto por el modelo Harmonie-Arome para la isla de Gran Canaria para el día 28 de febrero de 2018 a las 22 UTC. Se observa como la zona del aeropuerto se veía afectada por vientos medios de $40 \mathrm{kt}(74 \mathrm{~km} / \mathrm{h})$ con rachas de hasta $60 \mathrm{kt}(111 \mathrm{~km} / \mathrm{h})$.
HARMONIE-AEMET 28-02-2018 12z, pronóstico para el Miercoles 28-02-2018 $22 \mathrm{z}(\mathrm{H}+10)$

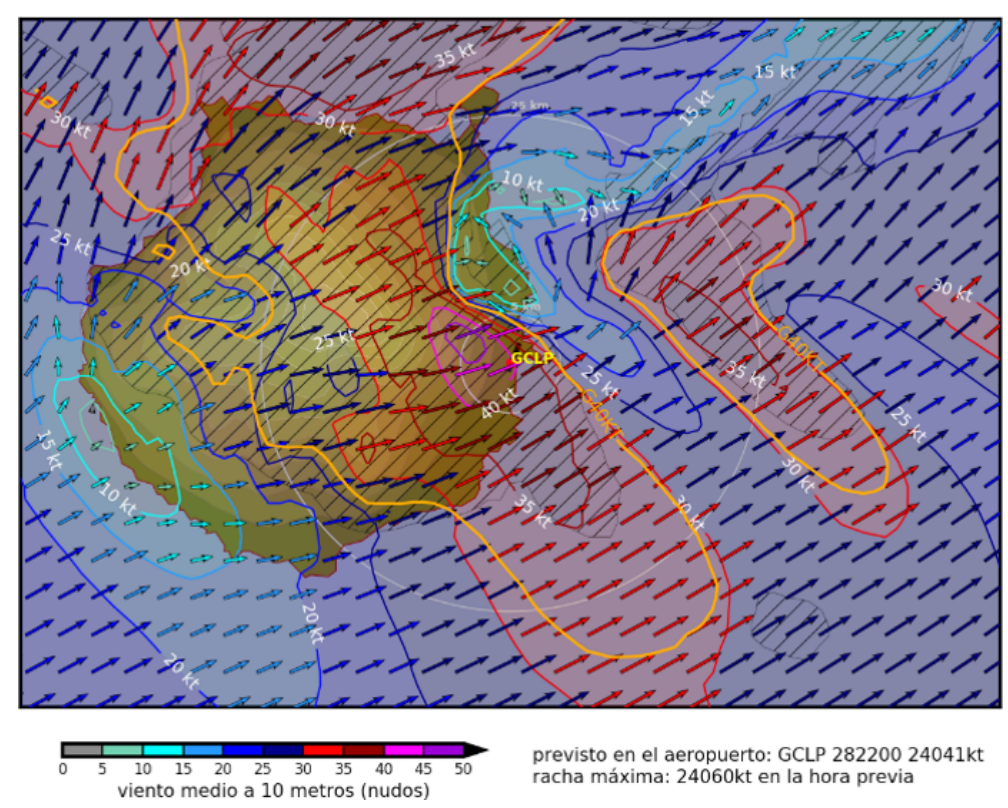

Figura 4. Viento a 10 metros (kt) previstos para la isla de Gran Canaria para el día 28 de febrero de 2018 a las 22 UTC (modelo Harmonie-Arome).
Viento (Har v40) Valido para 201704261000

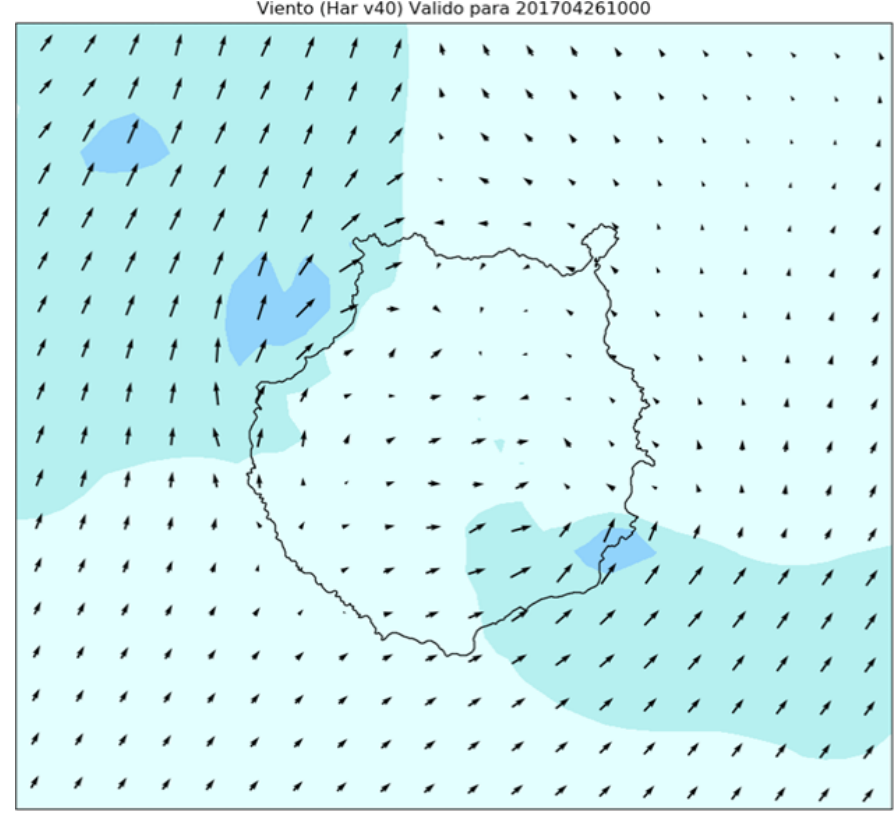

Figura 5. Viento a 10 metros $(\mathrm{kt})$ previstos para la isla de Gran Canaria para el día 26 de abril de 2017 a las 10 UTC (modelo Harmonie-Arome).

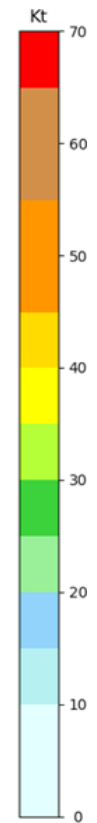

En la figura 5 se muestra el viento a 10 metros en nudos previstos por el modelo Harmonie-Arome para la isla de Gran Canaria para el día 26 de abril de 2017 a las 10 UTC. En este caso los vientos son en general débiles a moderados, inferiores a $20 \mathrm{kt}(37 \mathrm{~km} / \mathrm{h})$ y en la zona del aeropuerto se observa una cizalladura horizontal direccional debido al efecto de la superposición de las brisas con el viento del suroeste sinóptico.

La situación del 28 de febrero de 2018 fue, aparte de una situación muy severa, muy interesante, ya que a pesar de existir unas condiciones meteorológicas similares entre los aeropuertos de Gran Canaria y Tenerife Sur, este último no experimentó un elevado número de operaciones frustradas.

El origen de estas notables diferencias pudo estar relacionado con estructuras mesoescalares que afectaron de forma local al aeropuerto de Gran Canaria.

En la figura 6 se muestra una imagen de satélite RGB de masas de aire. En esta imagen se puede ver como la región de Canarias quedaba situada entre dos masas de aire marcadamente diferentes. Por un lado la masa de aire ártico marítimo, al norte de las islas, mostrada en la imagen por tonos azules, que representan aire frío con una tropopausa baja y, por otro lado, la masa de aire tropical continental, marcada en la imagen por tonos verdes, que denotan aire cálido con una tropopausa alta. 


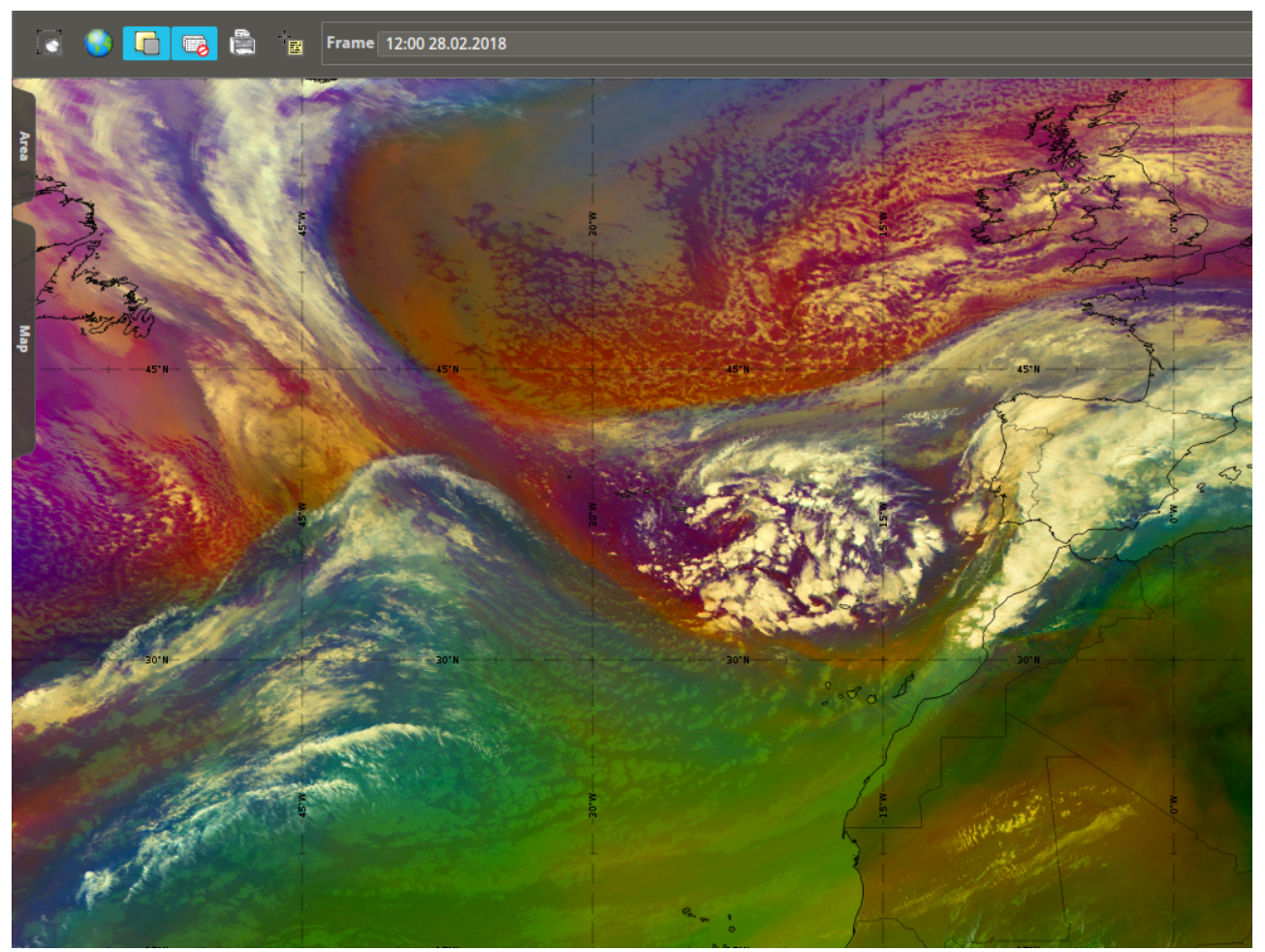

Figura 6. Imagen RGB de masas de aire para el día 28 de febrero de 2018 a las 12 UTC.

Ambas masas de aire confluían en Canarias y se formaron líneas de convergencias mesoescalares al sur de las islas que se desplazaron en dirección SW-NE.

En la imagen de satélite del canal visible en alta resolución(HRV) de la figura 7 se muestra remarcada en rojo una de las líneas de convergencia que en su desplazamiento hacia el nordeste impactó con la zona de aproximación y del propio aeropuerto de Gran Canaria ocasionando un número considerable de operaciones frustradas.

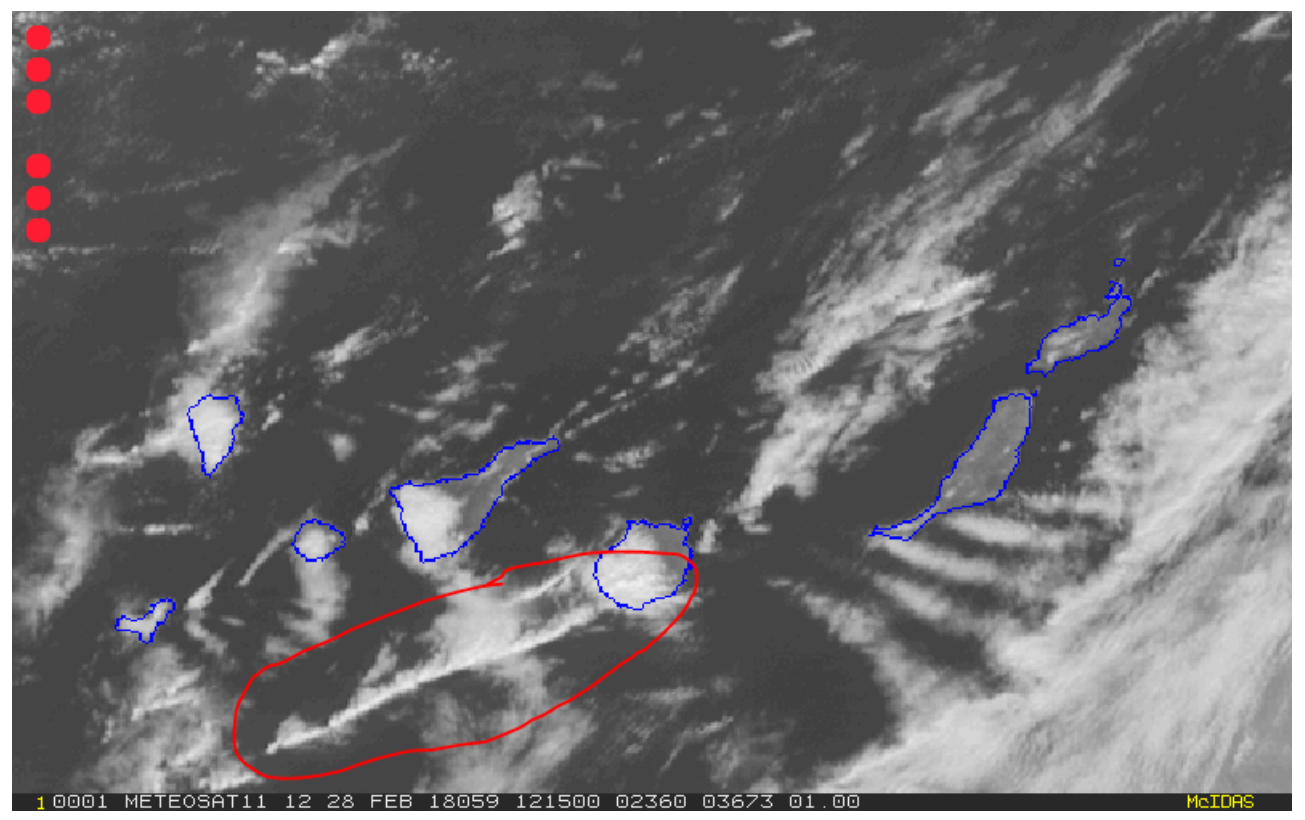

Figura 7. Imagen de satélite del canal HRV para el día 28 de febrero de 2018 a las 12:15 UTC. 


\section{PRODUCTOS EXPERIMENTALES DE PREDICCIÓN DE CIZALLADURA}

Se han creado productos experimentales específicos a partir de las salidas del modelo Harmonie-Arome para la predicción de cizalladura en los aeropuertos de Gran Canaria y Tenerife Sur. Para el cálculo de la magnitud de cizalladura se toman las componentes zonales y meridionales del viento y esta se calcula al nivel de interés según corresponda en función de la senda real de planeo establecida en cada aeropuerto. En la figura 8 se muestra una vista aérea del aeropuerto de Gran Canaria (GCLP), situado al este de la isla señalando las cabeceras.

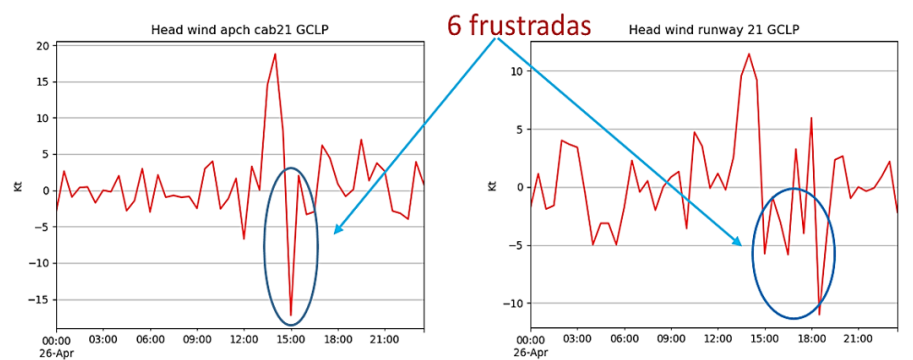

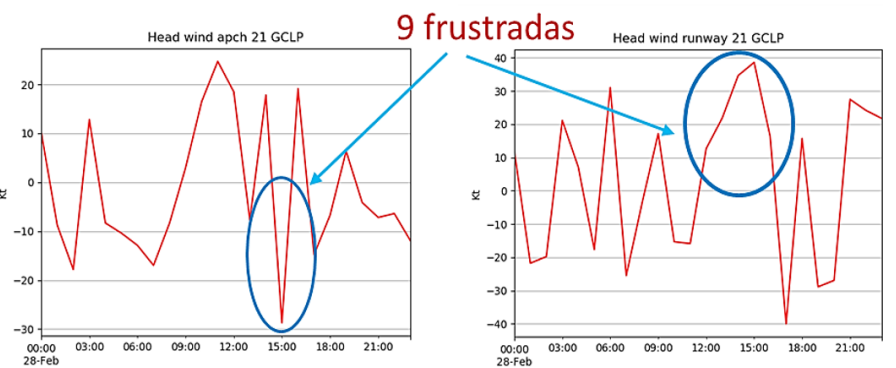

Figura 9. Serie temporal de viento de cara (head wind positivo) y viento de cola (head wind negativo) para los días analizados en aproximación y en la cabecera 21.
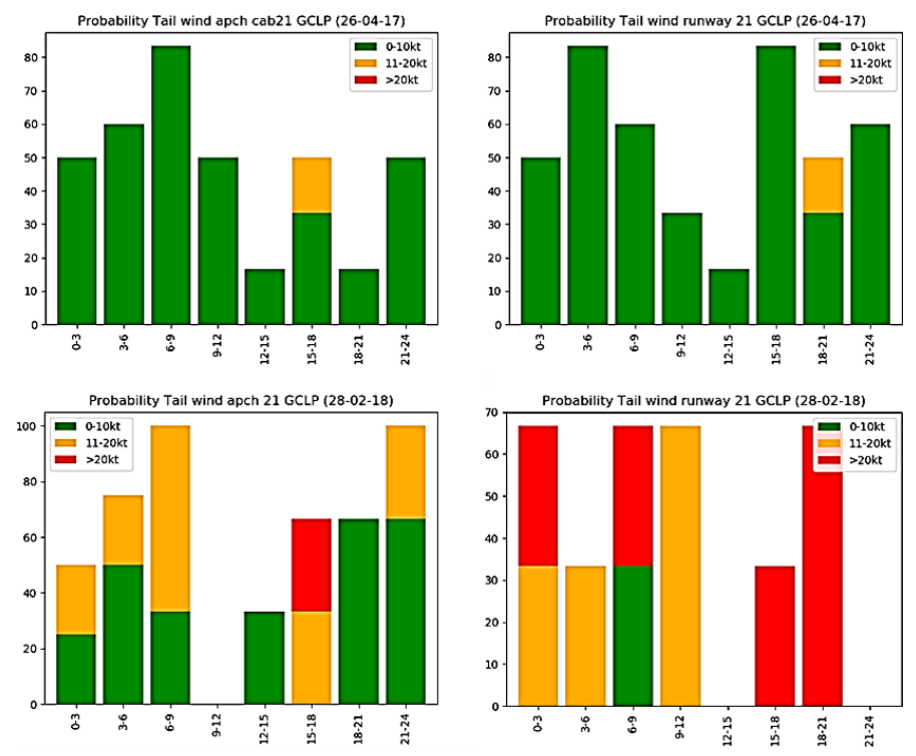

Figura 10. Probabilidad de viento de cola.

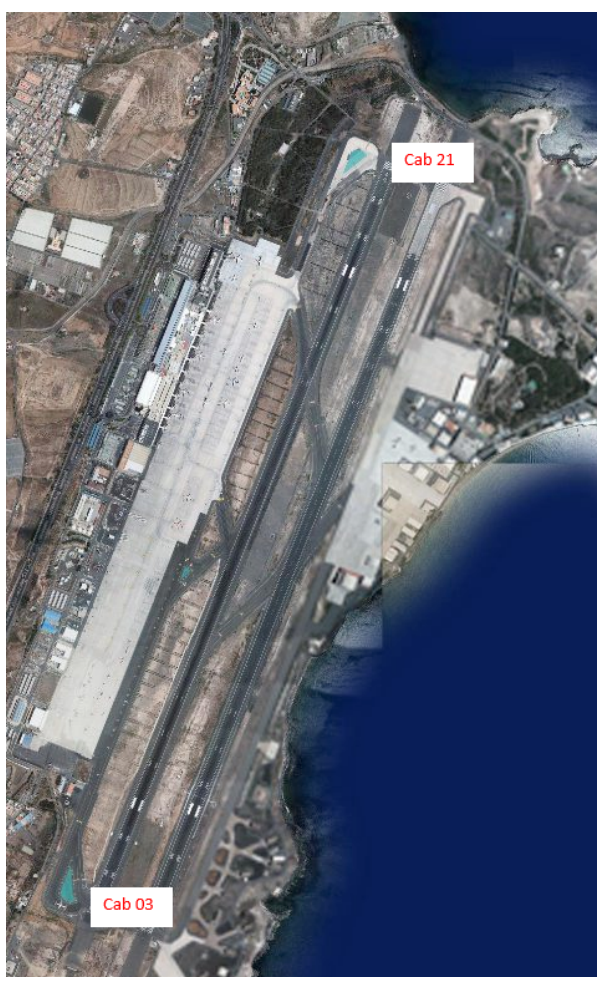

Figura 8. Vista aérea del aeropuerto de Gran Canaria.

En la fig. 9 se muestran varias series temporales de head wind (viento de cara) en las localizaciones denominadas como «apch $21 »$, esto quiere decir, aproximación a la cabecera 21 (que era la cabecera operativa ambos días analizados), y en la propia cabecera 21. El punto «apch 21» está localizado a 2 millas de la cabecera 21 y al nivel de $656 \mathrm{ft}$ (200 m).

En la fig. 10 se muestran varios gráficos que señalan la probabilidad que existe de encontrar tail wind (viento de cola) agrupada por horas en las localizaciones señaladas (tanto en «apch 21» como en la cabecera 21 del aeropuerto de GCLP). Además, en estos gráficos las barras tienen diferente color según la intensidad (del viento de cola en este caso).

En ambos episodios la cabecera operativa fue la 21, y los productos experimentales mostraban una alta probabilidad de viento de cola en aproximación y/o en pista, coincidiendo con un alto número de operaciones frustradas.

Además de los productos anteriores, desde el pasado mes de agosto se ha implantado un sistema 
experimental de detección automática de cizalladura (figura 11). El sistema está compuesto por dos subsistemas, basados ambos en metodologías diferentes. El primero de ellos está fundado en la emisión de auto-warnings cuando se produce un cambio sustancial del viento de cara en la senda de planeo de la aproximación final. El segundo subsistema emitirá un auto-warning cuando se supere un umbral (basado en percentiles 90, 95 y 99 de las series analizadas) de cizalladura total en la senda de planeo de la aproximación final.

Estos productos están en fase de prueba, verificación y calibración, así que no deben usarse de forma operativa ni extraerse conclusiones significativas en esta fase. Estos productos están disponibles únicamente de forma interna en la intranet de AEMET.

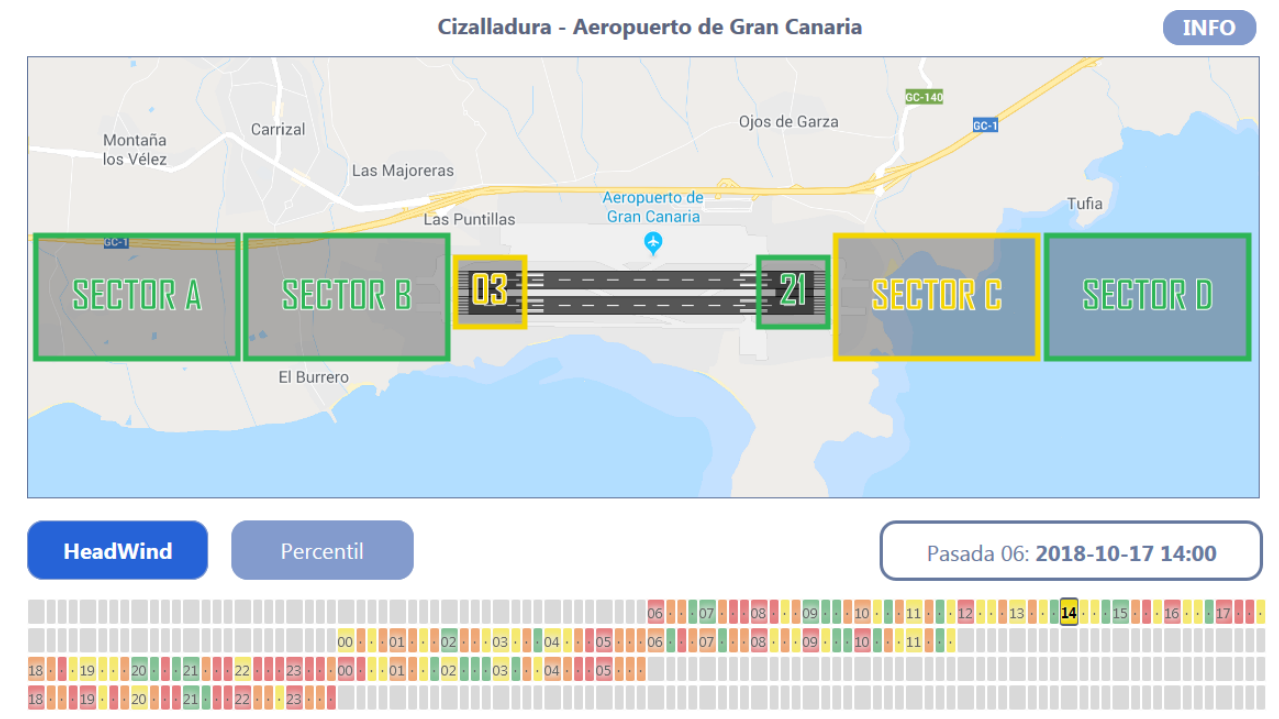

Figura 11. Visualización del sistema experimental de detección automática de cizalladura.

\section{IMPACTO}

Durante los dos episodios analizados (26 de abril de 2017 y 28 de febrero de 2018) se produjeron un total de 35 aterrizajes frustrados $(14+21$, respectivamente), con desvíos a aeropuertos alternativos y numerosas incomodidades para pasajeros y personal a bordo. A nivel operacional y de seguridad aérea, el principal problema fueron los vientos cruzados en aproximación, con cambios repentinos del viento de cola a morro e intensidades relativas superiores a los $60 \mathrm{kt}$ en el tramo entre Tufia (figura 12) y la propia cabecera 21 de GCLP. El impacto entre las aeronaves fue generalizado, viéndose afectados tanto turbohélices como reactores.

Desde el punto de vista del impacto económico para las aerolíneas, se produjeron pérdidas asociadas a episodios de aterrizajes frustrados puntuales (tipificados como aquellos en los que la aeronave logra aterrizar en un nuevo intento poco después, con un coste asociado al extra de combustible), y también a desvíos de parte del tráfico aéreo entrante, lo que supuso sobrecostes por pernoctaciones/pérdidas de conexiones del pasaje, nuevos repostajes, costes de handling o recambios en la tripulación de servicio por cese de actividad, entre otros.

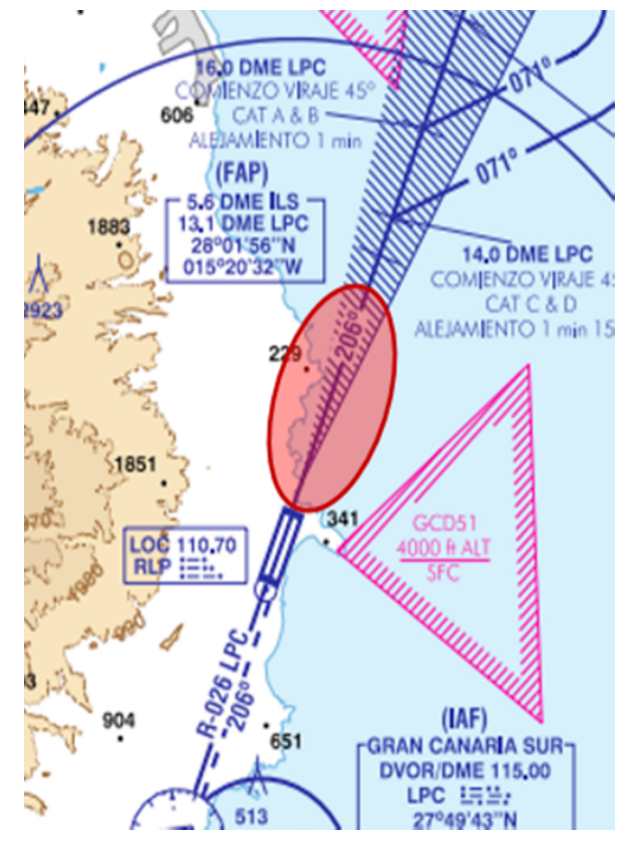

Figura 12. Zona donde se produjo la máxima cizalladura señalada sobre la carta aeronáutica. 


\section{CONCLUSIONES}

Este trabajo pone de manifiesto el impacto que produce la cizalladura en niveles bajos a las operaciones aeroportuarias de despegue y aterrizaje. Para la caracterización de la cizalladura en el aeropuerto de GCLP se han analizado más de 15000 aeronotificaciones de cizalladura y queda constatado que, en gran medida, este fenómeno es inducido por la compleja orografía de la isla de Gran Canaria.

Recientemente se han creado herramientas para el pronóstico de la cizalladura y se encuentran en fase de pruebas para verificación y calibración. Estas herramientas pueden ser de gran utilidad a la hora de mejorar la seguridad en las operaciones de aterrizaje y despegue de los aeropuertos al contar con horas de antelación de pronósticos que alerten sobre el riesgo de cizalladura.

\section{AGRADECIMIENTOS}

Este trabajo se ha realizado como parte del proyecto «Estudio de la cizalladura en el aeropuerto de Gran Canaria» enmarcado dentro del plan anual de aeronáutica 2018 de AEMET.

Los autores de este artículo quieren agradecer a todos los miembros del proyecto arriba mencionado, en especial a Olga Suárez Bravo de Laguna, jefa de la OMA (Oficina Meteorológica de Aeródromo) de Gran Canaria y miembro del proyecto, que ha aportado parte de la información incluida en este trabajo.

\section{REFERENCIAS}

Font, I., 1956. El Tiempo Atmosferico en las Islas Canarias. Servicio Meteorologico Nacional, 96 pp.

GonZÁLEZ, B., 2005. Meteorología aeronáutica. AVA.

Mazón, J., Rojas, J. I., Lozano, M., Pino, D., Prats, X. y Miglietta, M. M., 2018. Influence of meteorological phenomena on worldwide aircraft accidents, 1967-2010. Meteorol. Appl., 25 (2), 236-245.

Kessler, E., 1990. Low-level windshear alert systems and doppler radar in aircraft terminal operations. J. Aircr., 27 (5), 423-428. 